\title{
Radiation aging and chemi-crystallization processes in polyoxymethylene
}

\author{
B. Fayolle*, J. Verdu \\ Laboratoire PIMM, UMR 8006, CNRS - Arts et Métiers ParisTech, 151 Bd de l'Hôpital, 75013 Paris, France
}

Keywords:

Polyoxymethylene

Chain scission

Chemi-crystallization

Irradiation

\begin{abstract}
A B S T R A C T
The radiochemical degradation of a polyoxymethylene homopolymer (POM) was used to study the effects of molar mass changes in the crystalline structure. The dose rate was $20 \mathrm{kGy} \mathrm{h}^{-1}$ with doses of up to $30 \mathrm{kGy}$ used. Both WAXS and SAXS were used to analyse the structures. Results showed that, under irradiation, the polymer undergoes random chain scission. The radiochemical yield was found to be $G=1.6$ chain scission events per $100 \mathrm{eV}$. It was found that no crosslinking occurs and that only one chain scission mechanism, leading to the formation of formate groups, operates. Proof for the existence of chemi-crystallization is evidenced by (i) an increase in the crystallinity ratio as well as (ii) a decrease in the amorphous layer thickness. Simple models, derived from Rault's theory, are used to predict both (i) and (ii) from molar mass values.
\end{abstract}

\section{Introduction}

Although a semi-crystalline material always exists outside of a thermodynamic equilibrium, it reaches a quasi equilibrium when it is kept at a constant temperature $(T)$ above its glass transition temperature $\left(T_{\mathrm{g}}\right)$ and below its melting point $\left(T_{\mathrm{m}}\right)$. As a result of the existence of this quasi equilibrium, it was possible to establish suitable relationships between the molar mass distribution and characteristics such as the crystallinity ratio $\left(x_{C}\right)$ or the amorphous layer thickness $\left(l_{\mathrm{a}}\right)[1,2]$.

If a quasi equilibrated sample, which is kept at a particular temperature $(T)$, undergoes a random chain scission in its amorphous phase, it will undergo a secondary crystallization. This is often known as chemi-crystallization because it is induced by a chemical process. It can be assumed that, if the chain scission is slow enough, compared to crystallization, the morphology of the polymer can reach at any time the quasi equilibrium state characteristic of its new molar mass distribution.

From a practical point of view, chemi-crystallization can be important because it leads to serious embrittlement [3]. There are industrial application reasons, therefore, why

\footnotetext{
* Corresponding author.

E-mail address: bruno.fayolle@paris.ensam.fr (B. Fayolle).
}

it is not only interesting but also pertinent to establish the relevant relationships between the chemical variables (number of chain scissions, molar mass) and the morphological ones $\left(x_{\mathrm{C}}, l_{\mathrm{a}}\right.$, etc.).

Experimental data regarding chemi-crystallization are relatively abundant in the literature. Already in the early 1980 's, it was observed in the case of polyolefine oxidations [4-8] and poly(ethylene terephthalate) hydrolysis [9-11]. More recently there have been reports on polypropylene radiolysis [12,13] photooxidation [14]. In our early study involving the thermal oxidation of POM, chemi-crystallization was in fact observed [15]. However, although the occurrence of a depolymerization process did lead to the destruction of the amorphous phase and subsequently contributed to an increase in the crystallinity ratio, due to its complicated nature and the involvement of annealing effects, an accurate analyses of the chemi-crystallization kinetics proved too difficult. A more extensive study, using gamma ray irradiation at ambient temperature, specifically aimed at overcoming or at least minimizing these depolymerization and annealing effects is presented here.

It has been long established, that polymers which undergo thermal depolymerization i.e. polymers in which the monomer-monomer link is particularly weak, can undergo random chain scission when they are irradiated by ionizing radiations. Since POM belongs to this family of 
the polymers, which undergo facile (almost complete) thermal depolymerization, it is expected that on irradiation POM will undergo "pure" (no crosslinking) random chain scission. It is therefore a model polymer and a good candidate for investigating both chemi-crystallization mechanisms and kinetics.

\section{Experimental}

The POM used for this study was a homopolymer (Delrin 100) supplied by Dupont. Average molar masses were $M_{\mathrm{n}}=70 \mathrm{~kg} \mathrm{~mol}^{-1}, M_{\mathrm{W}}=140 \mathrm{~kg} \mathrm{~mol}^{-1}$ and polydispersity index $(\mathrm{PI})=2$. Glass transition temperature $\left(T_{\mathrm{g}}\right)$ of the amorphous phase was $-50^{\circ} \mathrm{C}$ and melting point $\left(T_{\mathrm{m}}\right)$ was $177^{\circ} \mathrm{C}$.

Samples of $4 \mathrm{~mm}$ thickness were injection moulded. Specific attention was paid to processing conditions in order to obtain quasi isotropic samples. The presence of a low degree of orientation was checked by SAXS (see below). The samples were annealed under vacuum $24 \mathrm{~h}$ at $130^{\circ} \mathrm{C}$ in order to reach quasi equilibrium before irradiation.

Gamma irradiation (60Co source) was performed in the SCK-CEN (Belgium) facility. The dose rate was $20 \mathrm{kGy} \mathrm{h}^{-1}$. Samples were exposed in air at $25^{\circ} \mathrm{C}$ to doses of up to 30 kGy.

Any modification in molecular structure was monitored using FTIR spectrophotometry, which followed the formate build-up, as well as molar mass measurements. The chemical changes and the homogeneity of the degradation was established using IR micro-spectrophotometry in transmission mode (Brucker IFS 28 with $4 \mathrm{~cm}^{-1}$ resolution). Samples consisted of transversal microtomed slices of $35 \mu \mathrm{m}$ thickness.

Rheometry in dynamic oscillatory mode at $190{ }^{\circ} \mathrm{C}$ under nitrogen was used to make molar mass measurements. An ARES rheometer (Rheometrics Scientific) in parallel plate configuration (diameter $25 \mathrm{~mm}$, gap $0.5 \mathrm{~mm}$ ) at atmospheric pressure in the $0.1-100 \mathrm{rad} \mathrm{s}^{-1}$ frequency range was used. The maximum strain amplitude was optimized in order to measure reliable torque values in the Newtonian domain. Weight average molar mass $\left(M_{\mathrm{W}}\right)$ was determined from Newtonian viscosity $(\eta)$ using the well-known scaling law $\eta=K M_{\mathrm{W}}^{3.4}$ with $K\left(190^{\circ} \mathrm{C}\right)=9.56 \times 10^{-15} \mathrm{SI}$. The molar mass distribution was determined from the viscoelastic spectrum using Mead's model [16] in order to assess polydispersity index and $M_{\mathrm{n}}$.

Wide-angle X-ray Scattering (WAXS) and Small-angle $\mathrm{X}$-ray Scattering (SAXS) were used to study the crystalline structure of the polyoxymethylene. This was achieved by means of a 2D diffraction system (Inel, France) equipped with a copper anode. The selected tension and the intensity were $30 \mathrm{kV}$ and $40 \mathrm{~mA}$, respectively. The $K_{\alpha 1}$ copper radiation $\left(\lambda \mathrm{Cu}_{\alpha 1}=0.154 \mathrm{~nm}\right)$ wavelength used was selected by means of a parabolic multilayer mirror (Osmic) and a very thin capillary. The 2D transmission patterns recorded on an image plate system were digitalized by means of a scanner with a resolution of 25 microns. The diffraction intensity was corrected using the Lambert equation $I=I_{0}$ exp $(\mu \cdot t / \cos (2 \theta)), t$ being sample thickness. The diffraction angle was $2 \theta$. In the cases studied, the absorption coefficient $\mu$ was $11.204 \mathrm{~cm}^{-1}$. [17] The samples were parallel pipedic with a thickness of $0.8 \mathrm{~mm}$. In the subsequent WAXS analysis, the Lorentz-corrected SAXS profiles, $I(2 \theta)$, were analyzed using PeakFit software (SPSS Inc.) with a view to extracting the different components: (i) background, (ii) crystalline peaks and (iii) amorphous halo. The weight crystallinity ratio $\left(x_{\mathrm{C}}\right)$ was calculated from the relative area of the crystalline peaks. Subsequently, the long period $\left(l_{\mathrm{p}}\right)$ was obtained from the corrected diffraction curve $I(2 \theta)$ after derivation using Bragg law.

\section{Results and discussion}

\subsection{Chain scission process}

From molar mass measurements, it was found that irradiation induces a decrease in both $M_{\mathrm{n}}$ and $M_{\mathrm{W}}$, without a concomitant change in the polydispersity index (PI) (see Fig. 1a). The fact that the PI remains equal to 2.0 indicates that a "pure" random chain scission process is occurring. When $M_{\mathrm{W}}$ is plotted as a function of the dose, as shown in Fig. 1a, it clearly decreases in a hyperbolic way.

These results can be interpreted on the basis of the classical theory for random chain scission and crosslinking processes [18]. If $s$ and $x$ are numbers of moles of chain scissions and crosslinks per mass unit in an initially linear polymer respectively, it can be written:

$\frac{1}{M_{n}}-\frac{1}{M_{n 0}}=s-x$

$\frac{1}{M_{W}}-\frac{1}{M_{W 0}}=\frac{s}{2}-2 x$

Crosslinking is expected to predominate if $x>s / 4$. In this case, the viscosity would continuously increase and would diverge at a gel point defined by:

$2 x_{g}-\frac{s_{g}}{2}=\frac{1}{M_{W 0}}$

With $x_{\mathrm{g}}$ and $s_{\mathrm{g}}$ are crosslinks and chain scission number at the get point respectively.

For $s / 4>x>0$, gelation would not occur but crosslinking would induce branching and this in turn would be responsible for the progressive disappearance of the Newtonian plateau in the curve viscosity as a function of shear rate. Here, there is no disappearance of the Newtonian plateau. On the contrary, it widens while Newtonian viscosity decreases. This behavior is clearly indicative of a large predominance of chain scission and a negligible crosslinking.

If crosslinking is negligible, Eqs. (1) and (2) simplify then as:

$\frac{1}{M_{n}}-\frac{1}{M_{n 0}}=s$

$\frac{1}{M_{W}}-\frac{1}{M_{W 0}}=\frac{s}{2}$

This leads to the following expression for the polydispersity index: 

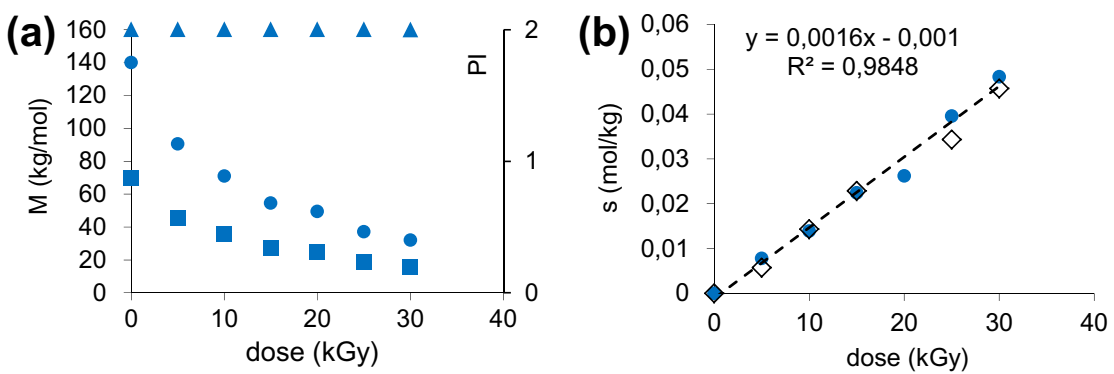

Fig. 1. (a) Weight average $\left(M_{\mathrm{W}}\right)(\boldsymbol{\bullet})$, number average $\left(M_{\mathrm{n}}\right)(\boldsymbol{\square})$ molar mass and polydispersity index ( $\left.\mathbf{\Delta}\right)$ as a function of dose, (b) chain scission number per mass unit $(\bullet)$ and number of formate groups per mass unit $(\diamond)$ as a function of the absorbed dose.

$I P=\frac{M_{W}}{M_{n}}=I P_{0} \frac{1+s M_{n 0}}{1+\frac{s}{2} I P_{0} M_{n} 0}$

According to Eq. (6), one sees that IP $\rightarrow 2$ when $s \rightarrow \infty$. The fact that the polydispersity index tends towards to 2 can be considered as a proof that chain scission occurs randomly. This property explains well why the polydispersity index of which the initial value is 2 , remains constant. It would have to increase in any case of significant crosslinking, non-random scission or heterogeneous degradation. for example, where $G(x)=2$ because it exclusively undergoes crosslinking under irradiation. In our case, the constancy of the PI values is strongly indicative of the absence of crosslinking, which is, of course, consistent with the known structure-radiochemical behavior relationships.

The only significant change in the IR spectrum in transmission mode is the growth of the band at $1736 \mathrm{~cm}^{-1}$.This can be attributed to the carbonyl stretching mode in formate groups. The presence of formates results most probably from the $\beta$ scissions of the macroradicals which in turn are a result of the radiochemical events:

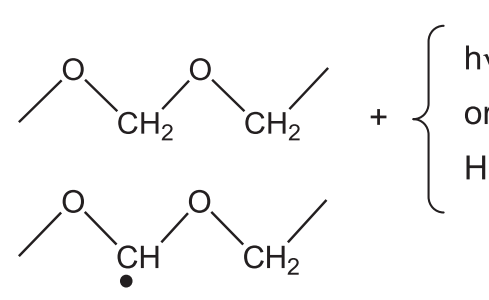

Eq. (5) has been used to determine the number $s$ of chain scissions. In Fig. 1b $s$ is plotted as a function of the dose and is seen to exhibit a quasi linear dependence:

$s \sim 16 \times 10^{-7} d$

where $s$ is in $\mathrm{mol} \mathrm{kg}^{-1}$ and $d$ in Gy.

The absence of auto-acceleration of the chain scission process in Fig. 1b indicates that oxidation is presumably negligible, that is not surprising for such relatively short exposures at room temperature $\left(25^{\circ} \mathrm{C}\right)$.

Using the unit classically used in the literature, the radiochemical yield of chain scission $G(s)$ is therefore found to be $16 \times 10^{-7}$ moles $\mathrm{J}^{-1}$ or 15 events $/ 100 \mathrm{eV}$. In his compilation of radiochemical yield values, Schnabel reported a value of $G(s)=11.1$ events $/ 100 \mathrm{eV}$ for POM [18]. This is significantly lower than our value but is still much higher than other common polymers that predominantly undergo depolymerisation. For example, for polyisobutylene $G(s)=1.5-5.0$ and for poly(methylmethacrylate) it is, 1.2-2.6 as well as 3.5-6.9 for cellulose [18]. Surprisingly, Schnabel reported a high value for the radiochemical yield of crosslinking $G(x)=6.5$. This is in contrast to polyethylene,
Microspectrophotometric FTIR mapping results (not shown) confirmed that the formates are uniformly distributed throughout the whole sample thickness. This indicates that chain scission results mainly from radiolysis events and that any eventual oxidation effects are negligible.

As shown in Fig. 1b, using the Beer Lambert law and a molar absorptivity value of $\varepsilon_{\mathrm{CO}}=500 \mathrm{~mol}^{-1} \mathrm{~cm}^{-1}$ for formate [15], their concentrations were determined and plotted as a function of the dose. It appears that the number of formates is equal, within experimental error, to the number of chain scissions determined from the molar mass measurements. In other words, from these results it can be safely assumed that no other chain scission mechanism other than the one above, are occurring.

\subsection{Crystallinity changes}

Irradiation-induced crystallinity changes can result, in principle, from two opposing phenomena: the creation of defects in the crystalline phase as characterized by the radiochemical yield $G$ (cryst), and chemi-crystallization. The $G$ (cryst) values are, however, relatively low [19] so that 


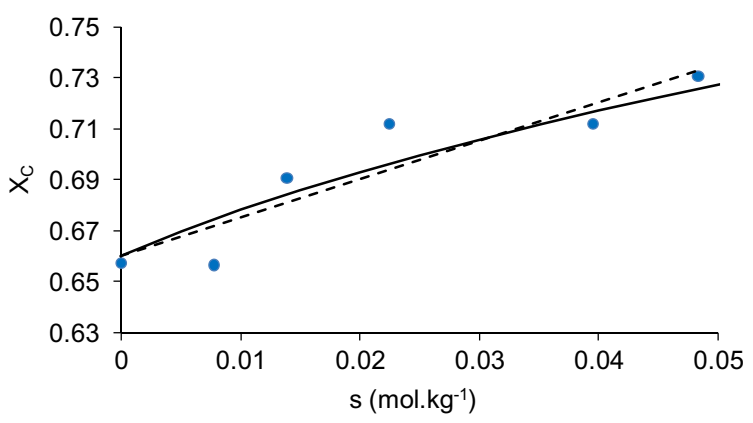

Fig. 2. Crystallinity ratio $\left(x_{\mathrm{C}}\right)$ as a function of chain scission number. Points correspond to experimental data, dashed line to linear fit. Curve to fit by Eq. (13) using $a=0.6$ and $b=0.71$ (see text).

their eventual effects become significant only at doses considerably higher than the maximum dose (30 kGy) employed here. Hence, it is safe to conclude that the crystallinity changes observed here are dominated by chemi-crystallization.

Since the WAXS measurements effectively reveal an increase in the crystallinity ratio $x_{C}$ this confirms both the presence and the predominance of chemi-crystallization. It seemed pertinent to plot the crystallinity ratio as a function of the number of chain scissions and these results are shown in Fig. 2 . It is clearly seen that $x_{C}$ increases almost linearly with $s$. This result leads to define the chemi-crystallization yield $Y$ as the number of monomer units entering the crystalline phase per chain scission:

$Y=\frac{1}{M_{m}} \frac{d x_{c}}{d s}$

where $M_{\mathrm{m}}$ is the molar mass of the monomer unit $\left(0.03 \mathrm{~kg} \mathrm{~mol}^{-1}\right)$.

The slope in Fig. 2 was determined to be $\mathrm{d} x_{\mathrm{C}}$ $\mathrm{d} s \sim 1.5 \mathrm{~kg} \mathrm{~mol}^{-1}$; so that the yield of chemi-crystallization is $Y \sim 50$ monomer units per scission event. The same value was found in the case of POM thermal oxidation at $130{ }^{\circ} \mathrm{C}$. [15] This indicates that $Y$ is not temperature dependant in the $20-130^{\circ} \mathrm{C}$ range. It should be noted that the entanglement molar mass of POM is $\sim 3 \mathrm{~kg} \mathrm{~mol}^{-1}$ [20] and that corresponds to 85 monomer units. It appears, therefore, that more than a half of entanglement strands integrates into the crystalline phase for every chain scission. As a reviewer pointed out, one can notice also that a chain segment 50 monomer long is roughly equivalent to one chain fold in the crystal.

In order to confirm these results the following hypotheses was proposed: the equilibrium crystallinity ratio value should depend only on the molar mass, irrespective of the mode of sample preparation, whether degradation or synthesis. In other words, degraded samples should be equivalent to virgin samples of the same molar mass.

In the case of the virgin samples, by using Rault's theory $[21,22]$, according to which crystallization is limited by the number of chain entanglements, it is expected that the crystallinity ratio should be linked to the weight average gyration ratio $r_{\mathrm{W}}$ by Eq. (9):

$x_{c}=1-\Psi\left(\frac{1}{r_{W E}}-\frac{1}{r_{W}}\right)$
Here $r_{\mathrm{W}}$ and $r_{\mathrm{WE}}$ are the respective weight average gyration radii for the polymer under consideration and for a polymer having its molar mass close to the entanglement threshold, respectively. $\Psi$ is a parameter which depends on both the chemical structure and crystallization conditions. According to Robelin Souffaché and Rault [21], the weight average gyration radius is proportional to the square root of an average molar mass $M_{\mathrm{r}}$ defined by:

$M_{r}^{1 / 2}=\Sigma w_{1} M_{1}^{1 / 2}$

where $w_{\mathrm{i}}$ is the weight fraction of the $i$ th species of which the molar mass is $M_{\mathrm{i}}$. The authors report that:

$M_{n}<M_{r}<M_{w}$

Considering that, here, no polydispersity change occurs during degradation, it can be considered, at least in a first approximation, that the gyration radius is proportional to the square root of the weight average molar mass and $\Psi$ is a constant for given quasi-equilibrium conditions. Therefore Eq. (9) can be transformed into Eq. (12):

$x_{c}=a+b M_{w}^{-1 / 2}$

where $a$ and $b$ depend on structure and sample preparation.

By combining Eqs. (5) and (12) it is possible to obtain Eq. (13):

$x_{c}=a+b \frac{\left(1+\frac{s}{2} M_{w 0}\right)^{1 / 2}}{M_{w 0}^{1 / 2}}$

On first inspection, this relationship appears to disagree with the linear relationship found in Fig. 2. However, the curve representing Eq. (13) with $a=0.60$ and $b=0.71$ has been plotted in Fig. 2 and it is practically undistinguishable from a linear relationship within data scatter. This leads to the observation that the chemi-crystallization yield $Y$ is not rigorously constant but must slightly decrease when the molar mass decreases. It could be imagined that a decrease of molar mass induces an increase of chain mobility which would be expected to favor chemi-crystallization while the opposite trend is observed here. This apparently paradoxical result can be explained as follows. First, there is no consideration of crystallization kinetics here i.e. chemicrystallization is regarded as a transition between two equilibrium states corresponding to two distinct molar mass values. As a result, the corresponding yield does not depend of molecular mobility but rather on the gap between both states from equilibrium and this gap is a decreasing function of the crystallinity ratio. For an oligomer lacking of entanglements, we would have $x_{\mathrm{C}}=1$ and the chemi-crystallization yield would be zero.

It should be noted that the initial crystallinity ratio varying with the initial molar mass $M_{\mathrm{W}}$ as shown in Eq. (14)

$x_{c 0}=a+\frac{b}{M_{w 0}^{1 / 2}}$

depends on the mode of sample preparation, especially on the crystallization temperature. It is, therefore, not possible to use Eq. (13) with a single pair of parameter values, 
for instance $a=0.60$ and $b=0.71$, in all the cases. On the other hand, it is not realistic in each case to experimentally determine $a$ and $b$. If, however, we keep in mind that $x_{C}$ is a linear function of $M_{\mathrm{W}}{ }^{-1 / 2}$, whatever the processing conditions, then Rault's theory offers a solution. As a matter of fact, two points of the straight-line $x_{\mathrm{C}}=f\left(M_{\mathrm{W}}{ }^{-1 / 2}\right)$ are already known: The point corresponding to the initial sample properties $\left(x_{\mathrm{C} 0}, M_{\mathrm{W} 0}{ }^{-1 / 2}\right)$ and the point corresponding to the entanglement threshold $\left(1, M_{\mathrm{E}}^{-1 / 2}\right)$ As a matter of fact, according to Rault's theory [21], in the absence of chain defects, entanglements are the unique cause for limiting crystallization, so that the coordinates of the point corresponding to the entanglement threshold must be $\left(x_{\mathrm{C}}=1, M_{\mathrm{W}}=M_{\mathrm{E}}\right)$. This is, indeed, an oversimplification but this hypothesis allows to assess the trends of the phenomenon. From these coordinates, the following straight line Eq. (15) can be thus determined:

$x_{c}=x_{c 0}+A\left[\left(\frac{M_{w 0}}{M_{w}}\right)^{1 / 2}-1\right]$

where

$A=\frac{1-x_{c 0}}{\left[\left(\frac{M_{w 0}}{M_{E}}\right)^{1 / 2}-1\right]}$

when $s M_{\mathrm{w}_{0}}<10$ then Eq. (15) can be approximated in Eq. (17):

$x_{c}=x_{c 0}+\frac{A M_{w 0}}{5} s$

Finally,

$Y=\frac{1}{M_{m}} \frac{d x_{c}}{d s}=\frac{A M_{w 0}}{5 M_{m}}$

Here, with $x_{\mathrm{C} 0}=0.65, \quad M_{\mathrm{W} 0}=140 \mathrm{~kg} \mathrm{~mol}^{-1}$ and $M_{\mathrm{E}}=3 \mathrm{~kg} \mathrm{~mol}^{-1}$, one obtains $Y \sim 57$, in good agreement with the experimental value $(Y=50)$.

Thus, Eq. (17) gives a good approximation of the change in the crystallinity ratio for a given decrease of weight average molar mass as long as there are reasonable conversions of the degradation process, typically less than 10 scissions par initial chain of molar mass $M_{\mathrm{wo}}$.

In conclusion, it should be noted that the yield of chemi-crystallization is a decreasing function of the initial crystallinity ratio and an increasing function of the initial weight average molar mass.

\subsection{Changes in lamellar dimensions}

It is now been well established that important mechanical (fracture) properties are governed by the lamellar dimensions. In order to determine these, SAXS experiments were performed on the relevant POM samples. In Fig. 3 a typical SAXS pattern is shown for the absorbed doses of 0,5 and $30 \mathrm{kGy}$. The absence of orientation can clearly be seen.

The long period $\left(l_{\mathrm{p}}\right)$ was determined from diffractograms and plotted against the number of chain scissions as shown in Fig. 4. It was found that $l_{\mathrm{p}}$ remained constant up to $s \sim 0.03-0.04 \mathrm{~mol} \mathrm{~kg}^{-1}$, i.e. $\sim 4-6$ scissions per initial chain of molar mass $M_{\mathrm{Wo}}$. It was then found to decrease slightly but significantly from 19 to $18.2 \mathrm{~nm}$ at high doses.

From $l_{\mathrm{p}}$ and $x_{\mathrm{C}}$, it was possible to calculate the amorphous layer thickness $l_{\mathrm{a}}$, which was plotted against the number of chain scissions as shown in Fig. 4b. As the number of chain scissions reaches $0.05 \mathrm{~mol} \mathrm{~kg}{ }^{-1}$ i.e. 7 scissions per initial chain of molar mass $M_{\mathrm{Wo}} l_{\mathrm{a}}$ decreases continuously from 5.5 to $3.9 \mathrm{~nm}$.

The results can be interpreted as follows: At the beginning of irradiation exposure and before the number of chains scissions per initial chain of molar mass $M_{\mathrm{W}}$ has reached $5 \pm 1$, the constancy of the long period is consistent with the hypothesis of exclusive lamellae thickening ( $l_{C}$ increase) at the expense of the amorphous phase [15]. This process requires a rearrangement of the lamellae throughout their whole thickness. This is possible thanks to the existence of cooperative motions within the crystalline phase ( $\alpha$ or $\alpha_{C}$ motions) [23]. Indeed, knowing that the onset of $\alpha_{C}$ motions for POM is close to $70{ }^{\circ} \mathrm{C}$ at $1 \mathrm{~Hz}$ [24], large scale reorganization in the crystal is possible in the time scale of irradiation.

At high conversions of the chain scission process, typically for more than $\sim 5$ scissions per initial chain of molar mass $M_{\mathrm{W} 0}$, the fact that the long period is found to decrease would be usually interpreted in terms of the formation of new lamellae in the amorphous layer thickness. However, the expected SAXS peak at low diffraction angles does not appear. Furthermore, the nucleation of new lamellae needs a large amorphous layer thickness [21] and this is a condition that is difficult to fulfill in the context of this study. Finally, the observed small decrease of $l_{\mathrm{p}}$ at doses up to $25 \mathrm{kGy}$ can also be explained. Under conditions of high degrees of degradation depolymerisation is no

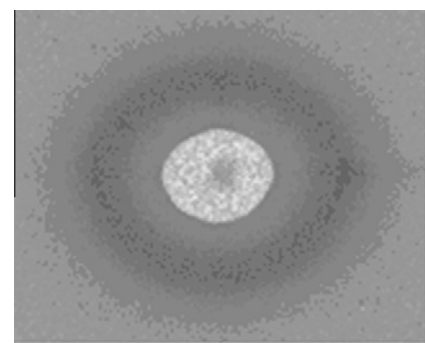

Ref

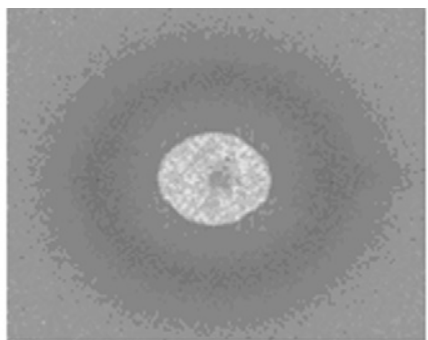

15 k Gy

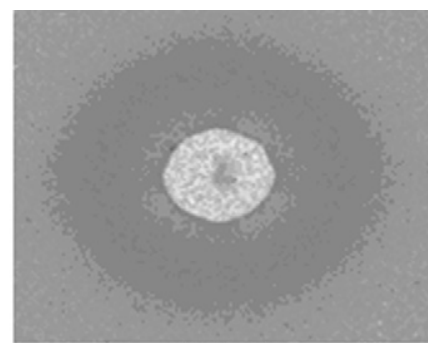

30 k Gy

Fig. 3. SAXS patterns at different absorbed doses. 

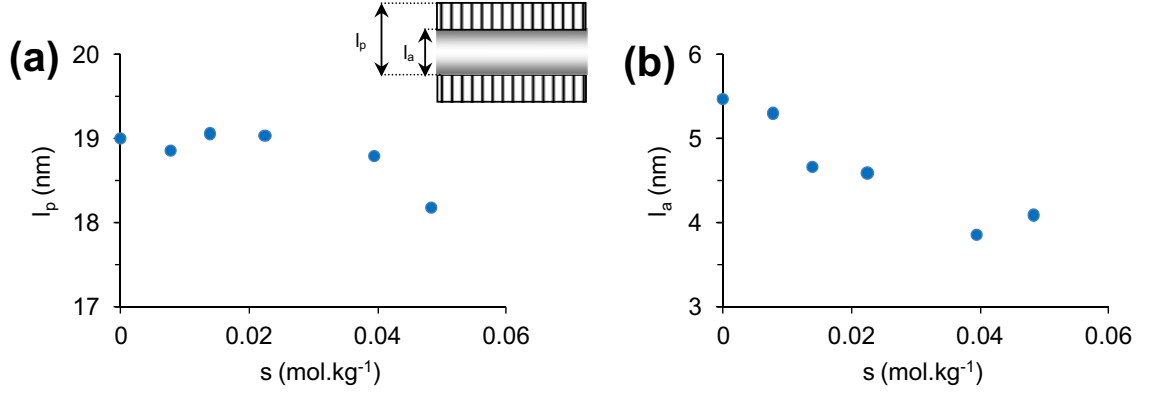

Fig. 4. (a) Long period $\left(l_{\mathrm{p}}\right)$ and (b) amorphous layer thickness $\left(l_{\mathrm{a}}\right)$ as function of the number of chain scissions.

longer negligible. Since a part of the amorphous phase disappears without any transfer to the crystalline phase, the sample is expected to shrink more than predicted as a result of crystallinity changes. Ultimately this shrinkage must lead to an affine decrease in the long period. It should be noted that this phenomenon is of secondary importance compared to the decrease in the amorphous layer thickness.

According to Rault's scaling law [21], there is also a linear relationship between $l_{\mathrm{a}}$ and the weight average gyration radius. Hence, using the same approximation as for Eq. (12), it is possible to write:

$\lambda_{a} \approx \lambda_{a 0}+c M_{w}^{1 / 2}$

With $c$ a constant.

The experimental value of the amorphous layer thickness was plotted against the square root of the weight average molar mass and the result is shown in Fig. 5. Within the molar mass range investigated, for a plot of the parameter values $l_{\mathrm{a} 0}=2.4 \mathrm{~nm}$ and $c=87 \times 10^{-4}, M_{\mathrm{W} 0}$ expressed in $\mathrm{g} \mathrm{mol}^{-1}$, the dependence appears to be correctly fitted by a straight line.

The fact that Eq. (19) is not rigorously consistent with Eq. (15), according to which $x_{\mathrm{C} 0}=0$, i.e. $l_{\mathrm{a}}=0$ for $M_{\mathrm{W}}=M_{\mathrm{E} .,-}$ could be raised. Indeed, as it is shown in Fig. 5, the full curve corresponding to Eq. (19) would presumably have the shape as indicated by the dashed line for low molar mass values.

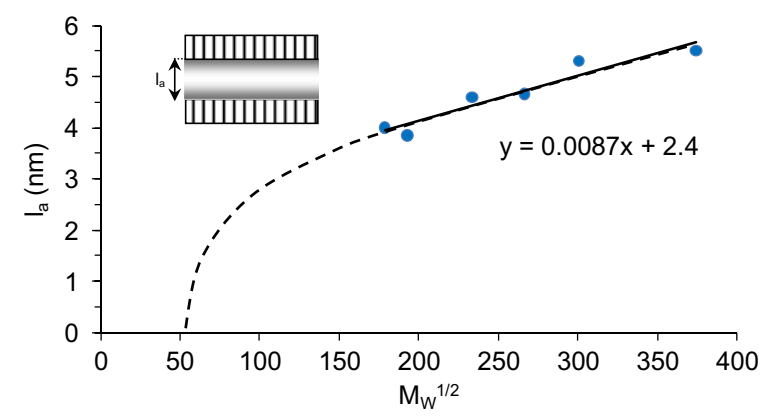

Fig. 5. Amorphous layer thickness $\left(l_{\mathrm{a}}\right)$ as a function of the square root of the weight average molar mass. The full line corresponds to the linear fit (Eq. (19)) of experimental points; the dashed line to the presumed real curve (see text).

\section{Conclusions}

Gamma ray irradiation using doses of up to $30 \mathrm{kGy}$ were chosen with the view to inducing random chain scission in POM. The experimental criteria were selected in order to avoid depolymerization and heterogeneity linked to an eventual kinetic control through oxygen diffusion. Rheometric and FTIR microspectrometry measurements confirm the absence of crosslinking, the random character of the chain scission as well as the homogeneity of the process throughout the sample volume. Analyses using WAXS and SAXS reveal the existence of a chemi-crystallization process in which both the crystallinity ratio $x_{C}$ and the amorphous layer thickness $l_{\mathrm{a}}$ vary continuously in order to adopt a quasi equilibrium value depending on the molar mass. The yield of chemi-crystallization is found to be about 50 monomer units per chain scission event. It is also confirmed that $x_{\mathrm{C}}$ increases almost linearly with $M_{\mathrm{W}}{ }^{-1 / 2}$ whereas $l_{\mathrm{a}}$ increases with its reciprocal $M_{\mathrm{W}}{ }^{1 / 2}$. Both trends are predicted by Rault's scaling law [21]. Using a very simple model derived from Rault's scaling law, it is possible to predict the yield of chemi-crystallization with good accuracy.

\section{Acknowledgements}

The authors wish to thank J.M. Hiver, A. Dahoun (Institut Jean Lamour, Nancy) and Aurélie Ollier for the DRX experimental part of the work.

\section{References}

[1] Kennedy MA, Peacock AJ, Mandelkern L. Macromolecules 1994;27:5297-310.

[2] Galeski A. Strength and toughness of crystalline polymer systems. In: Michler GH, Balta-Calleja FJ, editors. Mechanical properties of polymers based on nanostructure and morphology. Boca Raton, Florida: Taylor \& Francis; 2005. p. 159-211. Chap. 5.

[3] Fayolle B, Richaud E, Colin X, Verdu J. J Mat Sci 2008;43:6999-7012.

[4] Mucha M, Kryszewski M. Coll Polym Sci 1980;258:743-52.

[5] Wyzgoski MG. J Appl Polym Sci 1981;26:1689-704.

[6] Mathur Ajit B, Mathur GN. Polymer 1982;23:54-6.

[7] Gensler R, Plummer CJG, Kausch H-H, Kramer E, Pauquet J-R, Zweifel H. Polym Deg Stab 2000;67:195-208.

[8] Karlsson K, Smith GB, Gedde UW. Polym Eng Sci 1992;32:699-716.

[9] Miyagi A, Wunderlich B. J Polym Sci Part B: Polym Phys 1972;10:2073-83.

[10] Ellison S, Fisher LD, Alger KW, Zeronian SH. J Appl Polym Sci 1982;27:247-57.

[11] Ballara A, Verdu J. Polym Deg Stab 1989;26:361-74.

[12] Kostoski D, Stojanović Z. Polym Deg Stab 1995;47:353-6. 
[13] Zhang RC, Cameron RE. J Appl Polym Sci 1994;74:2234

[14] Rabello MS, White JR. Polym Deg Stab 1997;56:55-73.

[15] Fayolle B, Verdu J, Piccoz D, Dahoun A, Hiver JM, G'Sell C. J Appl Polym Sci 2009;111:469-75.

[16] Mead DW. J Rheol 1994;38:1797.

[17] Kakudo M, Kasai N. X-ray diffraction by polymers. Amsterdam, The Netherlands, New York, USA: Elsevier Publishing Company 1972.

[18] Schnabel W. In: Jellinek HG, editor. Aspect of polymer degradation and stabilisation. NY: Elsevier; 1978. p. 149-90. Chap. 4.
[19] Kellman R, Hill DJT, Hunter DS, O’Donnel JH, et Pomery PJ. ACS Symp Series 475, 119, ACS, Washington, 1991.

[20] Wu SJ. Polym Sci Part B: Polym Phys 1989;27:723-41.

[21] Rault J, Robelin-Souffaché E. J Polym Sci Part B: Polym Phys 1989;27:1349-73.

[22] Robelin-Souffache E, Rault J. Macromolecules 1989;22:3581-94.

[23] Strobl G. The physics of polymers. 2nd ed. Berlin: Springer; 1997. pp 248-255.

[24] Bastard M. Durability of polyoxymethylene. PhD Thesis ENSAM Paris, 2006. 